\title{
Sevoflurane preconditioning inhibits cardiomyocyte injury induced by oxygen-glucose deprivation by modulating TXNIP
}

\author{
MEINA MA, RUI LI, WENBO SUN, QI WANG, HONG YU and HONGMEI YU \\ Department of Anesthesiology, Cangzhou Central Hospital, Cangzhou, Hebei 061001, P.R. China
}

Received November 3, 2019; Accepted May 21, 2020

DOI: $10.3892 /$ ijmm.2020.4639

\begin{abstract}
The thioredoxin interaction protein (TXNIP) has been reported to be closely related to cell oxidative stress, apoptosis and inflammation. TXNIP is involved in the regulation of oxidative stress in lung and renal injury. However, it is unclear as to whether it participates in the protective effects of sevoflurane preconditioning in cardiomyocyte injury caused by oxidative stress in ischemia. In the present study, H9c2 cardiomyocytes were cultured with $0,1.5,2,3.5,5$ or $6 \%$ sevoflurane for $3 \mathrm{~h}$, followed by exposure to oxygen and glucose deprivation. The results demonstrated that oxygen and glucose deprivation induced an increase in TXNIP expression, lactate dehydrogenase (LDH) release, caspase-3 activity, reactive oxygen species and malondialdehyde production. Preconditioning of the H9c2 cells with $3.5 \%$ sevoflurane suppressed TXNIP expression, LDH leakage, caspase-3 activity, reactive oxygen species and malondialdehyde production, and it promoted cell viability. TXNIP overexpression reversed the effects of $3.5 \%$ sevoflurane preconditioning on caspase- 3 activity, reactive oxygen production and cell viability. Furthermore, TXNIP modulated p27 expression via PKB (protein kinase B/AKT) phosphorylation following preconditioning with $3.5 \%$ sevoflurane, and oxygen and glucose deprivation. On the whole these findings indicated that sevoflurane preconditioning protected the $\mathrm{H} 9 \mathrm{c} 2$ cells against injury induced by oxygen and glucose deprivation by modulating TXNIP, AKT activation and p27 signaling.
\end{abstract}

Correspondence to: Dr Meina Ma, Department of Anesthesiology, Cangzhou Central Hospital, 16 Xinhua West Road, Cangzhou, Hebei 061001, P.R. China

E-mail: mmeina1001@163.com

Abbreviations: TXNIP, thioredoxin interaction protein; AKT/PKB, protein kinase $\mathrm{B}$; OGD, oxygen-glucose deprivation; $\mathrm{LDH}$, lactate dehydrogenase; DCFH-DA, 2',7'-dichlorodihydrofluorescein diacetate; MTT, 3-(4,5-dimethyl-2-thiazolyl)-2,5-diphenyl-2-Htetrazolium bromide; MDA, malondialdehyde

Key words: sevoflurane preconditioning, oxygen-glucose deprivation, thioredoxin interaction protein, AKT, p27

\section{Introduction}

Myocardial ischemia is a major cause of cardiovascular disease, and acute myocardial ischemia is the most common form (1). Ischemia always gives rise to oxidative stress $(2,3)$. Studies have demonstrated that sevoflurane preconditioning can lessen myocardial oxidative stress and reduce myocardial damage $(4,5)$. Moreover, sevoflurane has been shown to reduce the myocardial infarct size in animal models and in mice with diabetes mellitus, as it protects the heart via AMPK-independent activation (6,7). Several randomized, controlled trials have demonstrated that sevoflurane reduces the levels of biomarkers of myocardial injury $(6,8)$. In rat models of myocardial ischemia-reperfusion, sevoflurane preconditioning has been shown to alleviate ischemia-reperfusion damage by inhibiting transcription factor SP1 (9). However, the molecular mechanisms of sevoflurane preconditioning during oxidative stress in cardiomyocytes remain unclear.

The thioredoxin interaction protein (TXNIP) is a small-molecule protein with redox activity $(5,10)$. TXNIP can bind to various proteins, such as Trx, and has various physiological functions, including regulating glucose metabolism and angiogenesis, inducing oxidative stress, and promoting apoptosis and inflammation (11-13). TXNIP plays a crucial role in ameliorating oxidative injury in diabetic kidneys (14). The inhibition of TXNIP suppresses oxidative stress and inflammation in lipopolysaccharide-induced acute lung injury (15). However, the role of TXNIP in the oxidative stress of cardiomyocytes remains unclear, and no available studies to date have evaluated whether TXNIP reduces oxidative stress following sevoflurane preconditioning in cardiomyocytes, at least to the best of our knowledge. In the present study, cardiomyocytes were preconditioned with sevoflurane, and were then exposed oxygen-glucose deprivation (OGD). Subsequently, the possible molecular mechanisms underlying the protective effects of sevoflurane against oxidative stress were investigated.

TXNIP exerts a regulatory effect on PKB (protein kinase $\mathrm{B} / \mathrm{AKT}$ ) phosphorylation in pancreatic $\beta$-cells, and AKT signaling can cause p27 downregulation in tumor cells $(16,17)$. AKT is a serine/threonine protein kinase that plays an important role in oxidative stress (18). AKT activation promotes curcumin-mediated resistance to oxidative stress in neurons (19). As a member of the cyclin-dependent kinase inhibitor family, p27 (p27kip1) regulates the cell cycle, apoptosis and cellular proliferation $(20,21)$, and it reportedly induces 
oxidative stress in liver cancer (22). The inhibition of oxidative stress is accompanied by a decreased p27 expression (23), and p27 has been demonstrated to protect cardiomyocytes from sepsis by suppressing apoptosis (24).

The effect of p27 on oxidative stress in cardiomyocytes remains unknown. It was hypothesized that TXNIP may regulate $\mathrm{p} 27$ by activating $\mathrm{AKT}$ and then modulating oxidative stress in cardiomyocytes that have been preconditioned with sevoflurane. The present study focused on identifying the role and molecular mechanisms of TXNIP by assessing the expression of TXNIP in cardiomyocytes following sevoflurane preconditioning. The data revealed that the downregulation of TXNIP protected H9c2 cells against injury induced by oxidative stress by modulating AKT and p27 following sevoflurane preconditioning.

\section{Materials and methods}

Cells and cell culture. H9c2 cardiomyocytes (ATCC) were cultured in Dulbecco's modified Eagle's medium (DMEM) supplemented with $10 \%$ fetal bovine serum, G418 $(80 \mu \mathrm{g} / \mathrm{ml})$ and hygromycin $\mathrm{B}(80 \mu \mathrm{g} / \mathrm{ml})$ in an incubator with $5 \% \mathrm{CO}_{2}$ at $37^{\circ} \mathrm{C}$. The sevoflurane preconditioning of the $\mathrm{H} 9 \mathrm{c} 2$ cells was achieved by culture with $0,1.5,2 \%, 3.5,5$ or $6 \%$ sevoflurane dissolved in DMEM for $3 \mathrm{~h}$. To inhibit AKT, H9c2 cells were incubated with $10 \mu \mathrm{M}$ of AKT inhibitor, LY294002 (S1105; Selleck Chemicals) for $12 \mathrm{~h}$.

$O G D$. H9c2 cells exposed to OGD were cultured with Earle's balanced salt solution in a humid atmosphere $\left(95 \% \mathrm{~N}_{2}\right.$ and $5 \% \mathrm{CO}_{2}$ ) for $4 \mathrm{~h}$ and then cultured with Earle's balanced salt solution supplemented with sugar in a non-hypoxic atmosphere $\left(95 \%\right.$ air and $\left.5 \% \mathrm{CO}_{2}\right)$ at $37^{\circ} \mathrm{C}$ for $9 \mathrm{~h}$. Subsequently, all media from the $\mathrm{H} 9 \mathrm{c} 2$ cells were changed to DMEM and cultured in $5 \% \mathrm{CO}_{2}$ at $37^{\circ} \mathrm{C}$ for $15 \mathrm{~h}$, as previously described (25). For indicated experiments, cells were preconditioned with $0,1.5,2,3.5,5$ or $6 \%$ sevoflurane for $3 \mathrm{~h}$ and then subjected to OGD.

Western blot analysis. Cells were treated with lysis buffer (Beyotime Institute of Biotechnology). Proteins were extracted and then quantified using a BCA kit (Perbio Science). Proteins $(25 \mu \mathrm{g})$ were loaded and separated in $10 \%$ sodium dodecyl sulfate polyacrylamide gel via electrophoresis. The separated proteins were electro-transferred to a polyvinylidene-fluoride membrane (Bio-Rad Laboratories, Inc.). The membrane was blocked with 5\% non-fat milk (Bio-Rad Laboratories, Inc.) in phosphate-buffered saline for $1 \mathrm{~h}$ and blotted with anti-TXNIP (1:800, sc-166234), anti-pan-AKT antibody (1:500, sc-5298), phosphor-AKT (Ser473; 1:800, sc-293125), and anti-p27 (1:800, sc-56338) antibodies (all from Santa Cruz Biotechnology Inc.). The membrane then was incubated overnight at $4^{\circ} \mathrm{C}$, followed by exposure to horseradish-peroxidase-conjugated secondary antibodies (1:1,000, sc-2005; Santa Cruz Biotechnology Inc.) for $1.5 \mathrm{~h}$ at room temperature. GAPDH was used as the internal control (1:2,000, sc-32233; Santa Cruz Biotechnology). The immunoblot signals were developed using an enhanced chemiluminescence kit (ECL kit, EMD Millipore) and the intensities of each band were measured by ImageJ 1.4.1 software (NIH).
MTT and lactate dehydrogenase ( $L D H)$ detection. Cells were cultured in a 96 -well cell plate $\left(1 \times 10^{5}\right.$ cells per well) in a humidified atmosphere of $5 \% \mathrm{CO}_{2}$ at $37^{\circ} \mathrm{C}$ for $12 \mathrm{~h}$ and then subjected to a 3-(4,5-dimethyl-2-thiazolyl)-2,5-diphenyl-2-H-tetrazolium bromide (MTT) assay. Briefly, the old medium was replaced with fresh medium plus $20 \mu \mathrm{l}$ of MTT solution $(0.5 \mathrm{mg} / \mathrm{ml})$ and incubated at $37^{\circ} \mathrm{C}$ for $5 \mathrm{~h}$. The formazan crystals were dissolved using $160 \mu \mathrm{l}$ of dimethyl-sulfoxide per well. Optical density was measured at $490 \mathrm{~nm}$. The solution was tested using a microplate analyzer model MR 600 (Dynatech Laboratories, Inc.). Using an LDH cytotoxicity detection kit (Takara Biotechnology, Inc.), the membrane integrity of the treated as was detected. Briefly, the transfected cells were incubated with chrysophanol for $24 \mathrm{~h}$, and LDH in the culture medium was detected according to the standard instruction.

Caspase- 3 activity assay. The caspase- 3 activity of the cells was measured in accordance with the instructions provided with the Caspase-3 activity assay kit (Shanghai Haoran Bio-Technology Co., Ltd.).

Measurement of reactive oxygen species (ROS). ROS release was determined using 2',7'-dichlorodihydrofluorescein diacetate (DCFH-DA) (Molecular Probes, Inc.) according to the specifications of the Reactive Oxygen Species Assay kit. Briefly, the cells were incubated with $60 \mu \mathrm{M}$ of DCFH-DA at $37^{\circ} \mathrm{C}$ for $45 \mathrm{~min}$ in the dark. Dichlorodihydrofluorescein fluorescence was determined using a flow cytometer (FACSCalibur; BD Biosciences) with an excitation wavelength of $485 \mathrm{~nm}$ and an emission wavelength of $530 \mathrm{~nm}$.

Detection of the malondialdehyde (MDA) concentration. The MDA concentration in the culture medium was quantified using MDA assay kits (Beyotime Institute of Biotechnology) according to the manufacturer's instructions. The absorbance was determined using an ELISA reader (MRX Microplate Reader, Dynatech Laboratories, Inc.) at $532 \mathrm{~nm}$.

Plasmid construction and cell transfection. The full-length DNA of the TXNIP gene (GenBank accession no. NM023719) and p27 gene (GenBank accession no. NM004064) were amplified from the cDNA of cardiomyocytes using PCR. The DNA was subsequently digested with restriction endonucleases EcoRI and BamHI and inserted into the pcDNA.3.1 (Invitrogen; Thermo Fisher Scientific, Inc.), which was cut with the same enzymes. Following transduction into Escherichia coli DH5 $\alpha$ (Gibco; Thermo Fisher Scientific, Inc.), the recombinant plasmid was amplified overnight at $37^{\circ} \mathrm{C}$. The plasmids were then sequenced, and the correct ones were designated as pcDNA.3.1-TXNIP and pcDNA.3.1-p27.

TXNIP C247S-pcDNA3.1 was obtained by in vitro mutagenesis using a Site Directed Mutagenesis kit (Beyotime Institute of Biotechnology), and full-length TXNIP-pcDNA3.1 was used as the template. All constructs were confirmed by sequencing.

For cell transfection, the cells were seeded into 24-well plates in a humidified atmosphere with $5 \% \mathrm{CO}_{2}$ at $37^{\circ} \mathrm{C}$, in accordance with the manufacturer's instructions for TurboFect (Thermo Fisher Scientific, Inc.). A total of 
$1.0 \mu \mathrm{g}$ of the pcDNA.3.1-TXNIP, pcDNA.3.1-p27, mutant pcDNA.3.1-TXNIP or pcDNA.3.1 were separately transfected into the cells with $2 \mu \mathrm{l}$ of TurboFect until cell fusion reached $80 \%$. The cells were then cultured in $5 \% \mathrm{CO}_{2}$ at $37^{\circ} \mathrm{C}$ for $24 \mathrm{~h}$.

Statistical analysis. Statistical analyses were performed using SPSS version 22.0 software (SPSS, Inc.). The Mann-Whitney $\mathrm{U}$ test was used to determine significant differences between 2 groups, and one-way ANOVA followed by a Bonferroni test was used for multiple groups. In all the figures, the data points and bar graphs represent the means of independent biological replicates. The error bars represent the standard deviation in graphs. A P-value $<0.05$ was considered to indicate a statistically significant difference. Each experiment was performed at least in triplicate in 3 independent experiments.

\section{Results}

Sevoflurane preconditioning increases cell viability, and inhibits apoptosis and LDH leakage in $\mathrm{H} 9 \mathrm{c} 2$ cells exposed to $O G D$. The H9c2 cells were cultured with $0,1.5,2,3.5,5$ or $6 \%$ sevoflurane in DMEM for $3 \mathrm{~h}$, followed by incubation under OGD conditions. Cell viability and LDH leakage were detected by an MTT assay and an LDH cytotoxicity detection kit, respectively. The results revealed that compared with the control (H9c2 cells without sevoflurane and OGD treatment), OGD induced a decrease in cell viability (Fig. 1A) and an increase in LDH leakage (Fig. 1B) and caspase-3 activity (Fig. 1C). However, preconditioning with sevoflurane at 3.5, 5 and $6 \%$ increased cell viability (Fig. 1A) and suppressed LDH leakage (Fig. 1B) compared to 0\% sevoflurane preconditioning. Caspase-3 activity also decreased by sevoflurane preconditioning at $3.5,5$ and $6 \%$ compared with $0 \%$ sevoflurane preconditioning (Fig. 1C).

Sevoflurane preconditioning reduces ROS production and the $M D A$ content in $H 9 c 2$ cells exposed to OGD. H9c2 cells were cultured under OGD conditions and then exposed to $3 \mathrm{~h}$ of preconditioning with $0,1.5,2,3.5,5$ or $6 \%$ sevoflurane. The data demonstrated that OGD induced an increase in ROS production (Fig. 2A) and the MDA content (Fig. 2B). However, sevoflurane preconditioning at $3.5,5$ and $6 \%$ significantly decreased the ROS levels (Fig. 2A) and the MDA content (Fig. 2B), compared with $0 \%$ sevoflurane preconditioning. Thus, sevoflurane preconditioning at $3.5 \%$ was selected for use in subsequent experiments.

Sevoflurane preconditioning inhibits TXNIP expression in $H 9 c 2$ cells exposed to OGD. The protein expression of TXNIP in the H9c2 cells exposed to sevoflurane preconditioning and OGD was assessed. It was found that the protein expression (Fig. 3A) of TXNIP increased following exposure to OGD compared with the control. However, TXNIP expression decreased in the H9c2 cells subjected to sevoflurane preconditioning compared with the cells not subjected to sevoflurane preconditioning.

TXNIP elevation promotes injury in $\mathrm{H} 9 \mathrm{c} 2$ cells exposed to sevoflurane preconditioning and OGD. TXNIP was overexpressed by transfection with pcDNA.3.1-TXNIP or a C247S

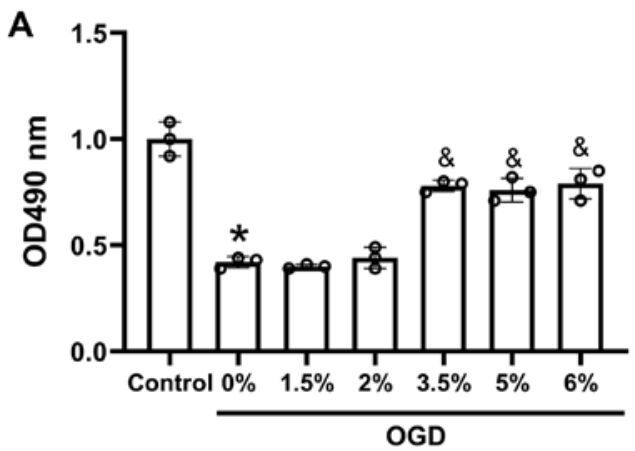

B

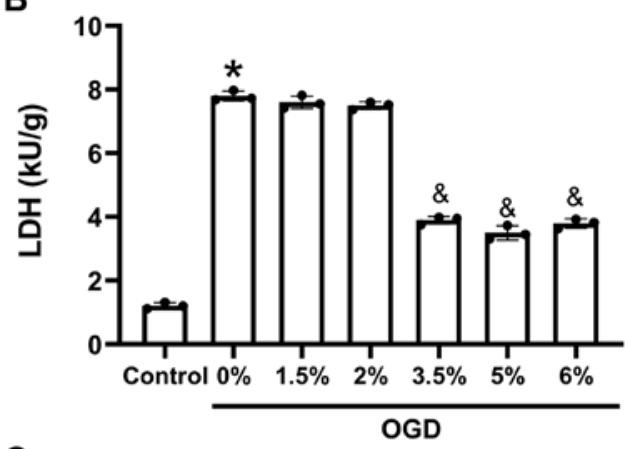

C

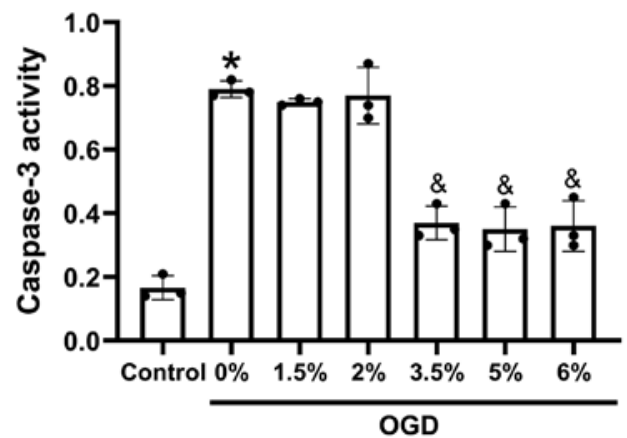

Figure 1. Effects of sevoflurane preconditioning on cell viability, lactate dehydrogenase leakage and apoptosis of H9c2 cells under exposed to OGD. (A) Cell viability following sevoflurane preconditioning and OGD, as measured by MTT assay. (B) LDH leakage following sevoflurane preconditioning and oxygen and glucose deprivation, as measured using an $\mathrm{LDH}$ cytotoxicity detection kit. (C) Apoptosis following sevoflurane preconditioning and OGD, as measured by caspase- 3 activity detection. H9c 2 cells were cultured in $0,1.5,2,3.5,5$ or $6 \%$ sevoflurane for $3 \mathrm{~h}$ and then exposed to OGD; $\mathrm{n}=3 ;{ }^{*} \mathrm{P}<0.01$ vs. the control group, ${ }^{\&} \mathrm{P}<0.05$ vs. the $0 \%$ group. OGD, oxygen-glucose deprivation; $\mathrm{LDH}$, lactate dehydrogenase.

TXNIP mutant plasmid under normal conditions or following sevoflurane preconditioning and OGD. The data indicated that the protein expression of TXNIP (Fig. 3B and C) following transfection was significantly upregulated in the H9c2 cells. It was also found that the overexpression of wild-type TXNIP or the C247S mutant TXNIP significantly decreased cell viability (Fig. 3D) and significantly elevated caspase-3 activity (Fig. 3E) and ROS levels (Fig. 3F).

TXNIP regulates $p 27$ by modulating AKT activation in $H 9 c 2$ cells exposed to sevoflurane preconditioning and OGD. AKT phosphorylation and p27 expression were measured following the overexpression of TXNIP in H9c2 cells subjected to sevoflurane preconditioning and OGD. The data demonstrated the elevated phosphorylation of AKT and the decreased 
A

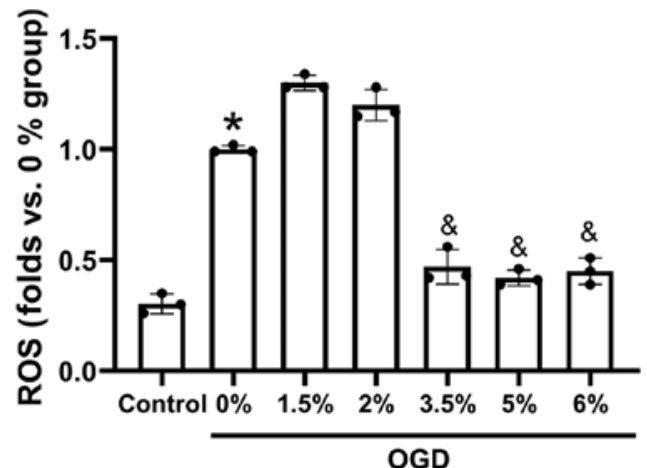

B

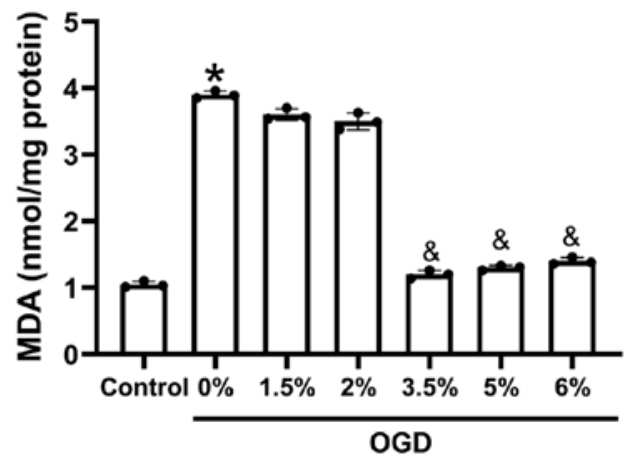

Figure 2. ROS and MDA contents following sevoflurane preconditioning in H9c2 cells exposed to OGD. (A) ROS and (B) MDA contents following sevoflurane preconditioning and OGD, as measured using a ROS assay kit and MDA assay kit, respectively; $\mathrm{n}=3$; ${ }^{*} \mathrm{P}<0.01$ vs. the control group, ${ }^{\text {\& }} \mathrm{P}<0.05$ vs. the $0 \%$ group. OGD, oxygen-glucose deprivation; MDA, malondialdehyde; ROS, reactive oxygen species.

A
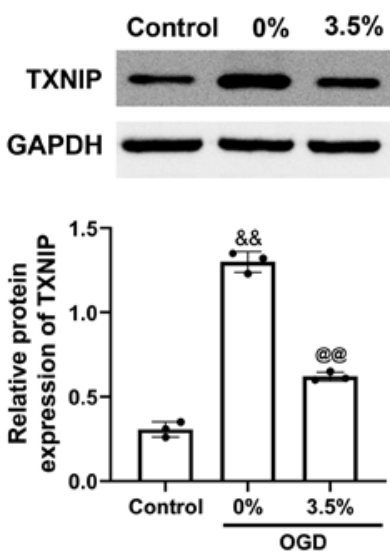

D

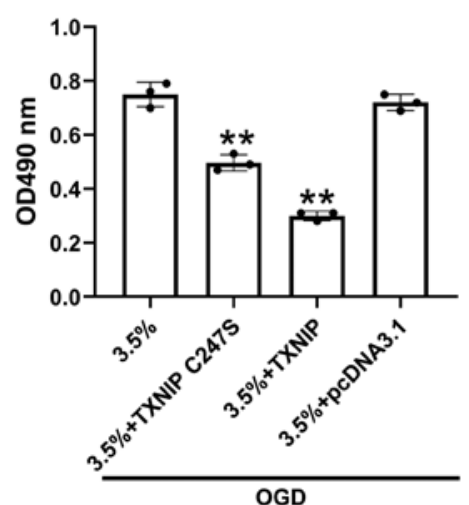

B
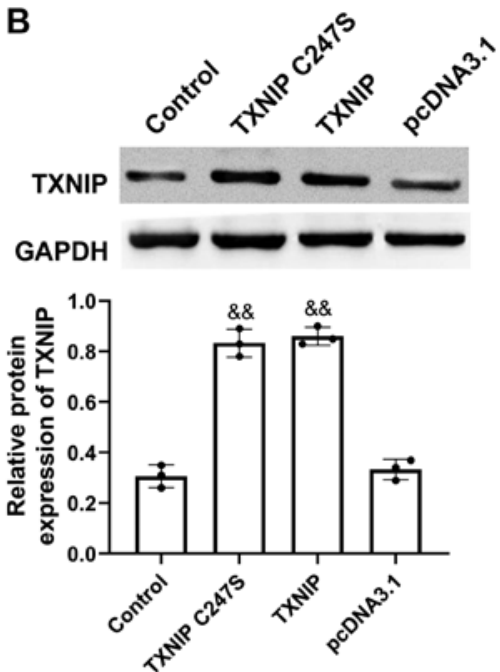

E

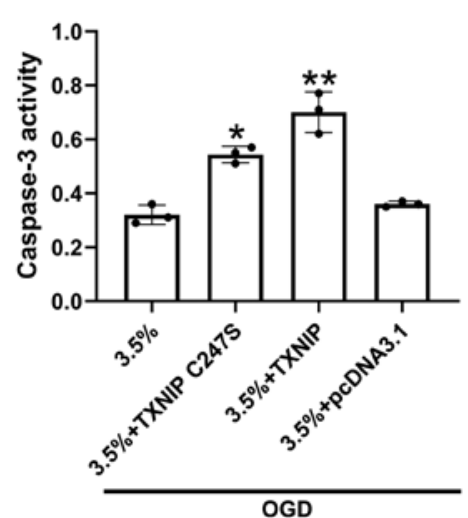

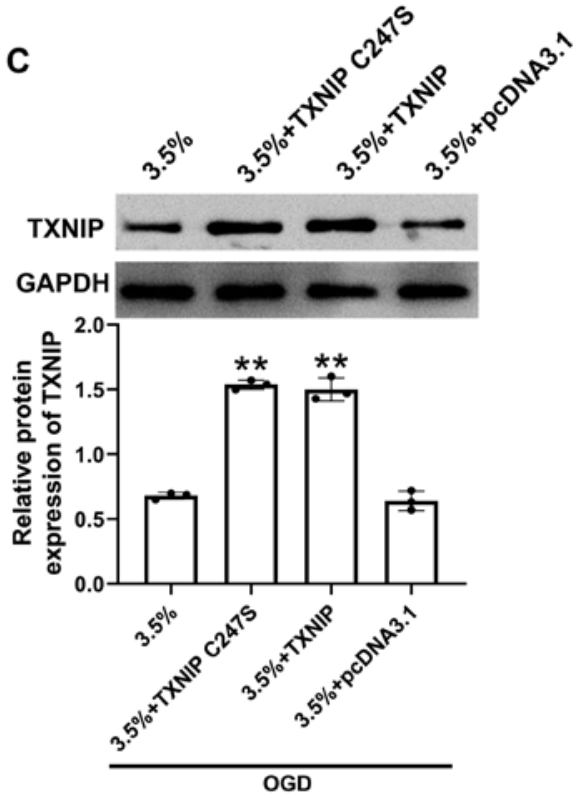

$\mathbf{F}$

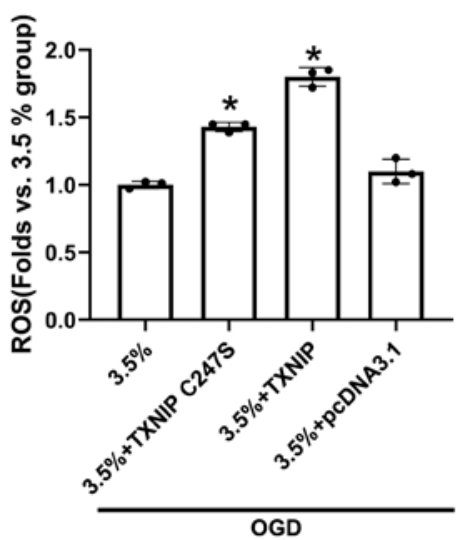

Figure 3. Effects of TXNIP upregulation on cell viability, apoptosis and ROS in H9c2 cells exposed to sevoflurane preconditioning and oxygen and glucose deprivation. (A) TXNIP protein expression in H9c2 cells with sevoflurane preconditioning and oxygen and glucose deprivation, as measured by western blot analysis. (B) Relative protein expression of TXNIP following transfection with pcDAN3.1-TXNIP in H9c2 cells under normal conditions or (C) in H9c2 cells subjected to sevoflurane preconditioning at 3.5\% and OGD measured by western blot analysis. (D) Cell viability following transfection in H9c2 cells subjected to sevoflurane preconditioning at 3.5\% and OGD, as measured by MTT assay. (E) Apoptosis following transfection of H9c2 cells subjected to sevoflurane preconditioning at 3.5\% and OGD, as measured by caspase-3 activity detection. (F) ROS levels following transfection of H9c2 cells subjected to sevoflurane preconditioning at 3.5\% and OGD, as measured using a ROS assay kit. 3.5\%+TXNIP, H9c2 cells transfected with pcDNA.3.1-TXNIP, TXNIP C247S mutant or empty plasmid pcDNA3.1 following sevoflurane preconditioning at 3.5\% and OGD; 3.5\%+pcDNA.3.1, H9c2 cells transfected with pcDNA.3.1 following sevoflurane preconditioning at $3.5 \%$ and OGD; $\mathrm{n}=3,{ }^{\& \&} \mathrm{P}<0.01$ vs. the control group, ${ }^{@} @ \mathrm{P}<0.01$ vs. the $0 \%$ group; ${ }^{*} \mathrm{P}<0.05$ and ${ }^{* *} \mathrm{P}<0.01$ vs. the $3.5 \%+$ pcDNA.3.1 group. TXNIP, thioredoxin interaction protein; OGD, oxygen-glucose deprivation; ROS, reactive oxygen species. 

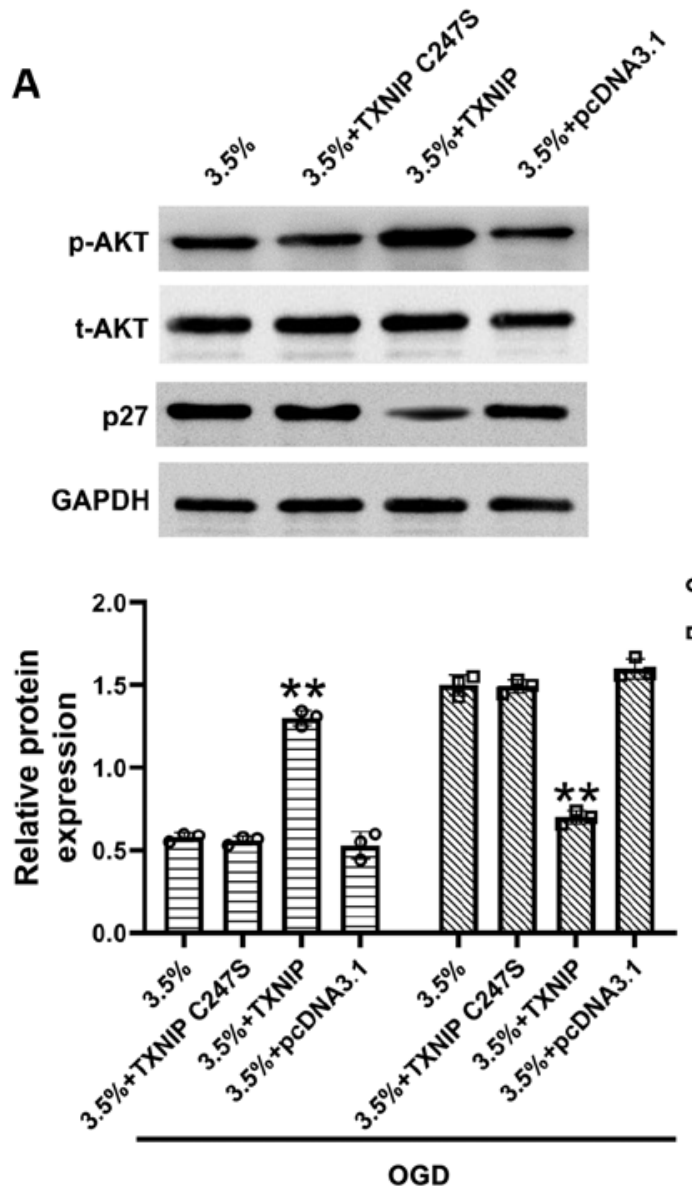

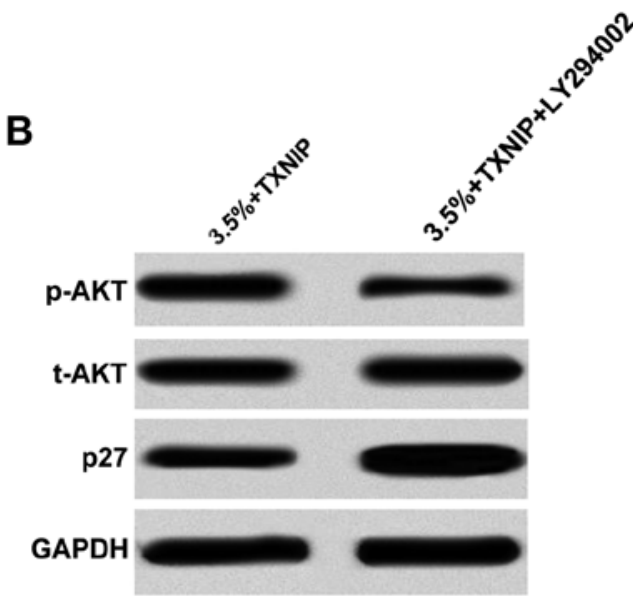

- p-AKT P27

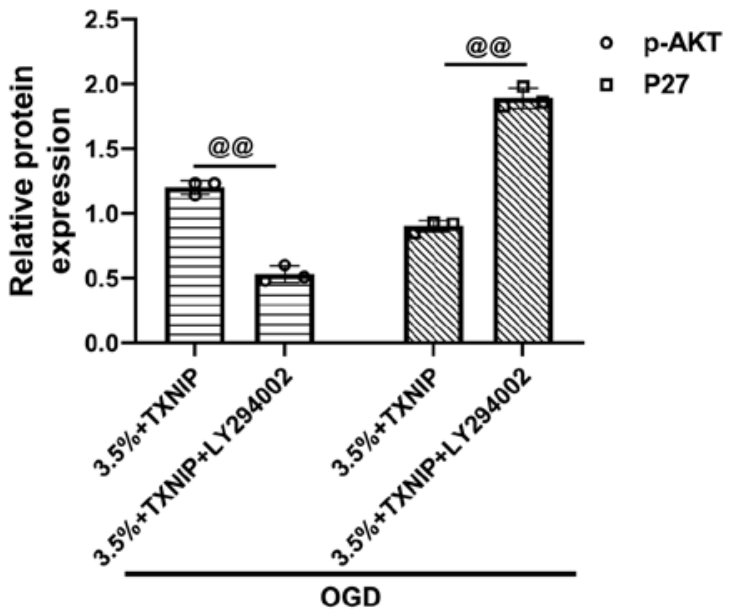

Figure 4. TXNIP regulates p27 expression via p-AKT in H9c2 cells subjected to sevoflurane preconditioning and OGD. (A) Protein expression levels of p27 and p-AKT after TXNIP elevation in H9c2 cells subjected to sevoflurane preconditioning at 3.5\% and OGD, as measured by western blot analysis. (B) Protein expression levels of p27 and p-AKT after TXNIP elevation and p-AKT inhibition in H9c2 cells subjected to sevoflurane preconditioning at 3.5\% and OGD, as measured by western blot analysis. 3.5\%+TXNIP+LY294002, H9c2 cells was incubated with LY294002 (10 $\mu \mathrm{M})$ and transfected with pcDNA.3.1-TXNIP, TXNIP C247S mutant or empty plasmid pcDNA3.1 following sevoflurane preconditioning at $3.5 \%$ and OGD; $n=3 ;{ }^{* *} \mathrm{P}<0.01$ vs. the $3.5 \%+$ pcDNA.3.1 group; ${ }^{\circledR @} \mathrm{P}<0.01$ vs. the $3.5 \%+\mathrm{TXNIP}$ group. TXNIP, thioredoxin interaction protein; OGD, oxygen-glucose deprivation.

expression of p27 following the overexpression of wild-type TXNIP (Fig. 4A), while the overexpression of the C247S TXNIP mutant exhibited no interaction with AKT (Fig. 4A). Incubation with the AKT inhibitor, LY294002 (10 $\mu \mathrm{M})(26,27)$, suppressed AKT phosphorylation and upregulated p27 expression (Fig. 4B), indicating that the inhibition of AKT activation reversed the effects of TXNIP on p27 expression in these cells. Thus, TXNIP regulates $\mathrm{p} 27$ expression via AKT activation in H9c2 cells exposed to sevoflurane preconditioning and OGD.

TXNIP expression mediated by sevoflurane preconditioning protects $H 9 c 2$ cells against injury induced by $O G D$ by modulating p27 expression. The p27 transfection efficiency was examined by western blot analysis. The results revealed that compared with the pcDNA3.1-transfected cells, the level of p27 was markedly increased in the p27-transfected H9c2 cells (Fig. 5A). In the H9c2 cells or H9c2 cells with sevoflurane preconditioning and OGD exposure, p27 overexpression was induced by pcDNA.3.1-p27 transfection following the overexpression of TXNIP. It was found that compared with the TXNIP- and pcDNA3.1-co-transfected cells, the level of p27 was markedly increased in the TXNIP-and p27-co-transfected
H9c2 cells, indicating the successful overexpression of p27 (Fig. 5B). In addition, p27 protein expression increased in the H9c2 cells subjected to sevoflurane preconditioning and OGD exposure (Fig. 5C). The upregulation of p27 promoted cell viability (Fig. 5D), and inhibited caspase-3 activity (Fig. 5E) and ROS production (Fig. 5F) to a greater extent in TXNIP-overexpressing H9c2 cells subjected to sevoflurane preconditioning and OGD injury than in H9c2 cells subjected to sevoflurane preconditioning and OGD exposure only. These results indicated that sevoflurane preconditioning protects H9c2 cells against injury induced by OGD by modulating TXNIP, AKT activation and p27 signaling (Fig. 6).

\section{Discussion}

Myocardial ischemia induces oxidative stress and widespread damage to cells (28-30). Sevoflurane preconditioning may protect the heart against this type of injury $(31,32)$. In the present study, cells were incubated with $0,1.5,2,3.5$, 5 or $6 \%$ sevoflurane and then exposed to OGD. The data indicated that sevoflurane preconditioning at 3.5\% markedly inhibited caspase-3 activity, LDH leakage, and MDA and 
A
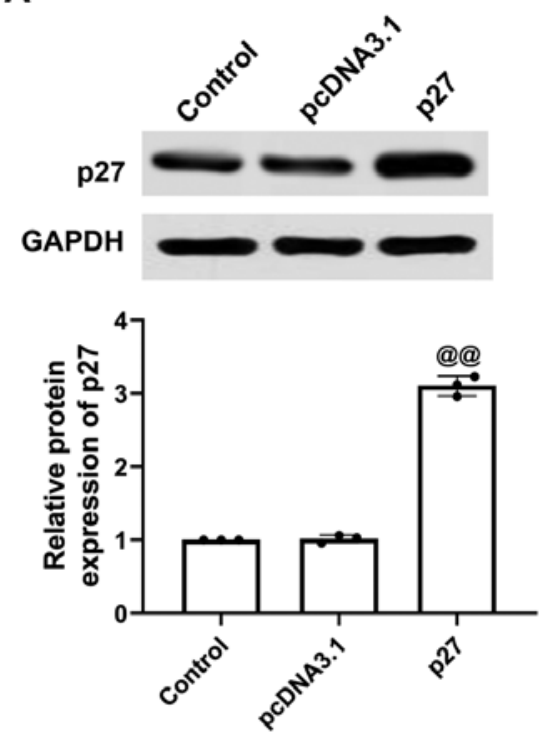

D

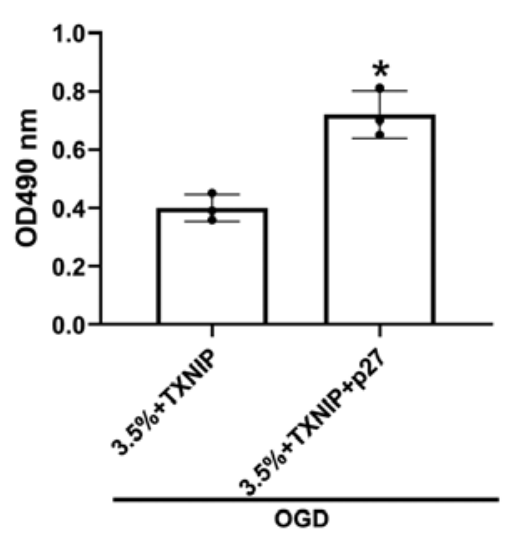

B
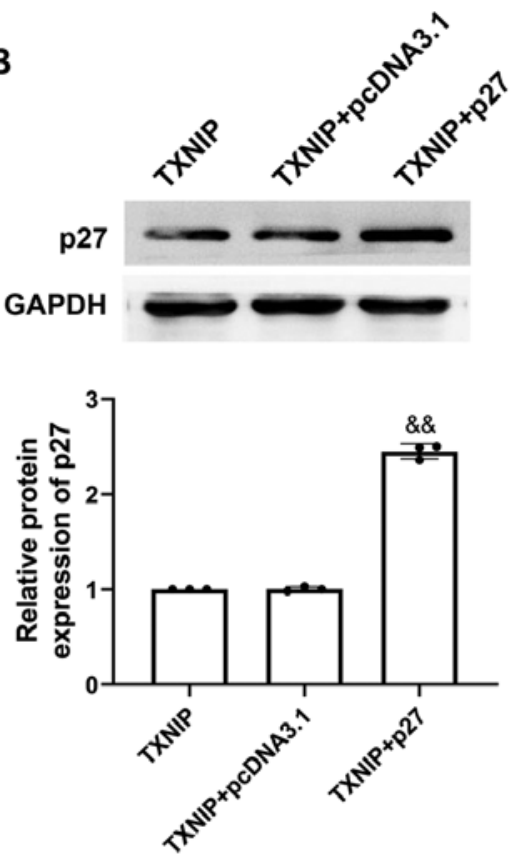

E

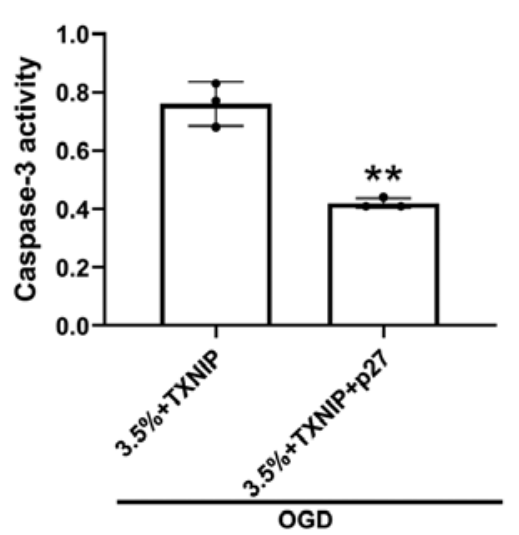

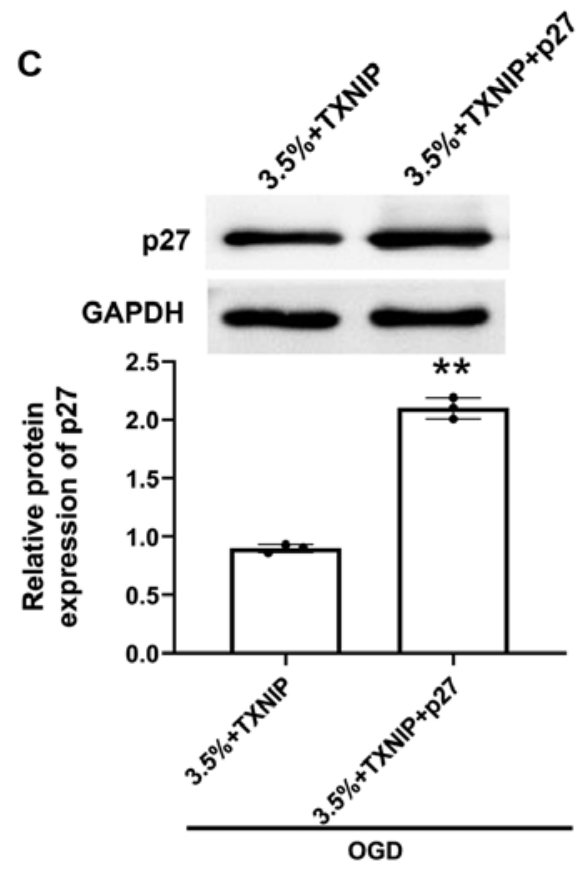

$\mathbf{F}$

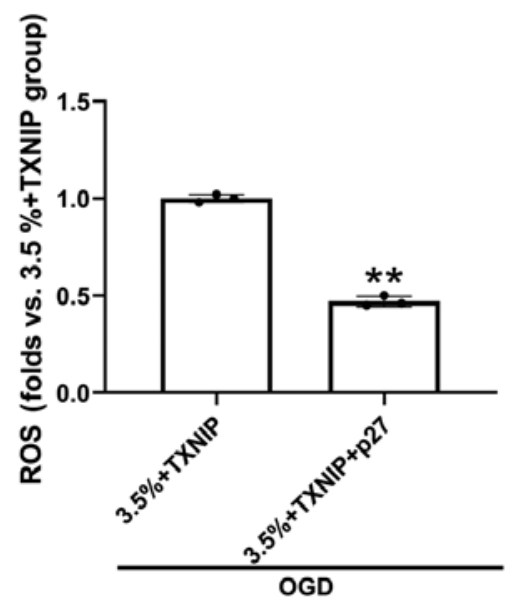

Figure 5. TXNIP regulates cell viability, apoptosis and ROS production via p27 in H9c2 cells subjected to sevoflurane preconditioning and OGD. (A) Relative protein expression of p27 following transfection with pcDNA3.1-p27 in H9c2 cells, as measured by western blot analysis. (B) Relative protein expression of p27 following transfection with pcDNA.3.1-p27 and pcDNA.3.1-TXNIP in H9c2 cells under normal conditions or (C) in cells subjected to sevoflurane preconditioning at 3.5\% and OGD as measured by western blot analysis. (D) Cell viability following the overexpression of TXNIP and p27 in H9c2 cells subjected to sevoflurane preconditioning at 3.5\% and OGD, as measured by MTT assay. (E) Apoptosis following the overexpression of TXNIP and p27 in H9c2 cells subjected to sevoflurane preconditioning at 3.5\% and OGD, as measured by caspase-3 activity detection. (F) ROS levels following the overexpression of TXNIP and p27 in H9c2 cells subjected to sevoflurane preconditioning at 3.5\% and OGD, as measured using a ROS assay kit. 3.5\%+TXNIP+p27, H9c2 cells transfected with pcDNA.3.1-TXNIP and pcDNA.3.1-p27 following sevoflurane preconditioning at 3.5\% and OGD; $=3$; ${ }^{@}{ }^{\circ} \mathrm{P}<0.01 \mathrm{vs}$. pcDNA3.1 group; ${ }^{\&}$ P $\mathrm{P}<0.01$ vs. the TXNIP+pcDNA3.1 group; ${ }^{*} \mathrm{P}<0.05$ and ${ }^{* *} \mathrm{P}<0.01$ vs. the $3.5 \%+\mathrm{TXNIP}$ group. TXNIP, thioredoxin interaction protein; OGD, oxygen-glucose deprivation; ROS, reactive oxygen species.

ROS production, and increased cell viability. The molecular mechanisms underlying the protective effects of sevoflurane preconditioning were then investigated.

TXNIP plays a role in salidroside-mediated protection against high-glucose-induced oxidative stress in rat glomerular mesangial cells (33). Hou et al demonstrated that the inhibition of TXNIP suppressed lipopolysaccharide-induced oxidative stress and the apoptosis of vascular endothelial cells (34). TXNIP overexpression has also been shown to induce cardiomyocyte apoptosis and injury (35). Currently, to the best of our knowledge, there is no available study to date on the role of TXNIP in the OGD-induced oxidative stress of cardiomyocytes following sevoflurane preconditioning. In the present study, the results indicated that sevoflurane preconditioning at $3.5 \%$ suppressed TXNIP expression in cardiomyocytes exposed to OGD. Furthermore, the overexpression of TXNIP by pcDNA.3.1-TXNIP transfection significantly increased caspase- 3 activity and ROS production, and it decreased the viability of cardiomyocytes subjected to sevoflurane preconditioning at $3.5 \%$ and OGD exposure. Previous studies have reported that $\mathrm{N}$-methyl-D-aspartic acid (NMDA) receptor is an important target for analgesia of sevoflurane (36). In addition, the blockade of NMDA receptor has been shown to dephosphorylate Forkhead box O1 (FOXO1) at Thr24 and induce its nuclear translocation, thus increasing the transcription of TXNIP (37). NMDA receptor blockade upregulates TXNIP, 


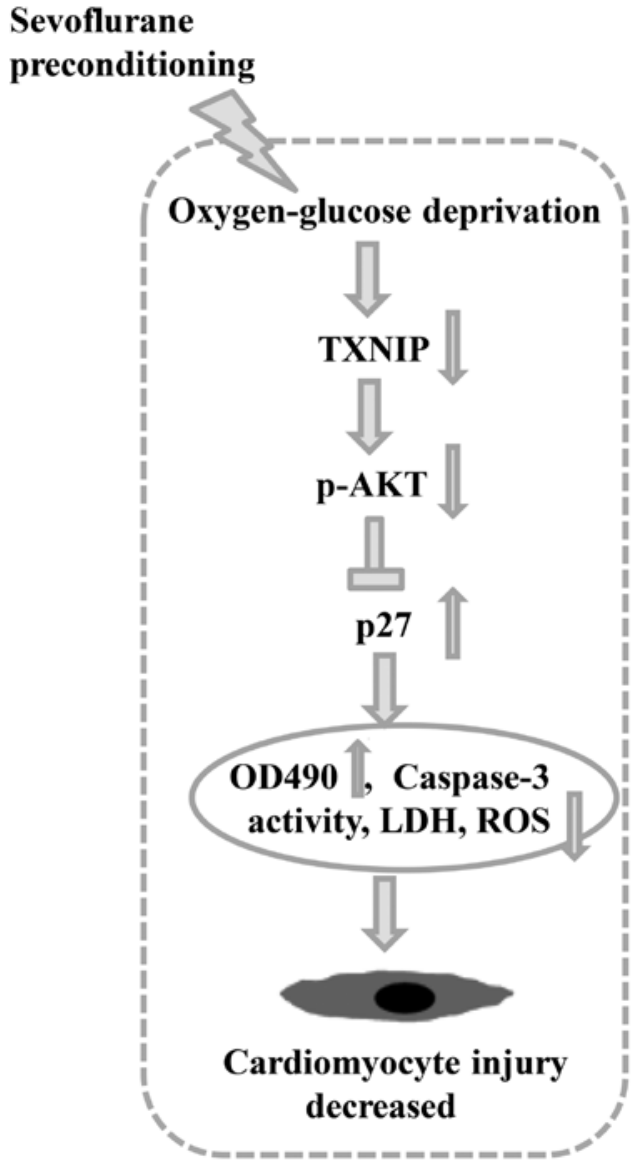

Figure 6. Schematic diagram depicting that sevoflurane preconditioning protects $\mathrm{H} 9 \mathrm{c} 2$ cells against injury induced by oxygen-glucose deprivation by modulating TXNIP, AKT activation and p27 signaling. Sevoflurane preconditioning inhibits TXNIP expression in H9c2 exposed to OGD, leading to a downregulation of p-AKT, while an upregulation of p27 in H9c2 cells, as well as the amelioration of cardiomyocyte injury. TXNIP, thioredoxin interaction protein; LDH, lactate dehydrogenase; ROS, reactive oxygen species.

where it binds thioredoxin and boosts antioxidant defenses via the thioredoxin-peroxiredoxin system (38). Thus, it was hypothesized that sevoflurane inhibits TXNIP expression through the NMDA receptor and FOXO1. The present study, to the best of our knowledge, is the first to demonstrate that the downregulation of TXNIP is closely related to the protective effects of sevoflurane preconditioning against oxidative stress in cardiomyocytes.

TXNIP regulates AKT phosphorylation in pancreatic $\beta$-cells, and AKT signaling can downregulate p27 in tumor cells $(16,17)$. Mahmoud et al demonstrated that AKT assists in the prevention of lipid-induced endothelial damage and oxidative stress (39). Zeng et al found that AKT is crucial in alleviating $\mathrm{H}_{2} \mathrm{O}_{2}$-induced oxidative stress caused by thyroid hormones in cardiomyocytes (40). In addition, p27 reportedly lessens the toxic effects of $\beta$-amyloid 42 during oxidative stress (41). Zhao et al demonstrated that p27 protects cardiomyocytes from sepsis by inhibiting apoptosis (24).

The effects of p27 on oxidative stress in cardiomyocytes subjected to sevoflurane preconditioning and OGD exposure remain unclear, however. It was hypothesized that TXNIP would regulate OGD-induced oxidative stress in cardiomyocytes subjected to sevoflurane preconditioning via AKT/p27 signaling. In the present study, TXNIP overexpression elevated the phosphorylation of AKT and decreased the expression of p27 in H9c2 cells exposed to sevoflurane preconditioning and OGD. The suppression of AKT phosphorylation by LY294002 $(10 \mu \mathrm{M})$ markedly increased the expression of $\mathrm{p} 27$ in $\mathrm{H} 9 \mathrm{c} 2$ cells with TXNIP overexpression. The results indicated that TXNIP regulated p27 expression via AKT in cardiomyocytes exposed to sevoflurane preconditioning and OGD. Moreover, p27 overexpression induced by pcDNA.3.1-p27 promoted cell viability and inhibited caspase- 3 activity and ROS production, indicating that $\mathrm{p} 27$ overexpression abolished the effects of TXNIP on cell viability, caspase- 3 and ROS in H9c2 cells subjected to sevoflurane preconditioning and OGD exposure. Therefore, it can be concluded that following sevoflurane preconditioning, sevoflurane protected cardiomyocytes against OGD-induced oxidative stress by regulating TXNIP/p27 expression.

In conclusion, the present study found that sevoflurane preconditioning at $3.5 \%$ significantly inhibited TXNIP expression, caspase- 3 activity, LDH leakage, and MDA and ROS production, and it increased the viability of $\mathrm{H} 9 \mathrm{c} 2$ cells that were exposed to OGD. TXNIP upregulation effectively reversed the effects of sevoflurane preconditioning at $3.5 \%$ on caspase- 3 activity, ROS production and cell viability. Moreover, the results indicated that TXNIP regulated $\mathrm{p} 27$ expression via AKT. Thus, sevoflurane preconditioning may protect cardiomyocytes against oxidative stress, and this effect may be modulated by TXNIP, AKT and p27 signaling (Fig. 6).

\section{Acknowledgements}

Not applicable.

\section{Funding}

No funding was received.

\section{Availability of data and materials}

The analyzed data sets generated during the study are available from the corresponding author on reasonable request.

\section{Authors' contributions}

All authors (MM, RL, WS, QW, HongY and HongmeiY) participated in the design, interpretation of the studies and analysis of the data and review of the manuscript. MM designed, prepared and performed the experiments. RL, WS, QW, HongY and HongmeiY contributed to the provision of reagents/materials/analysis tools. MM wrote the manuscript, and modified and revised the manuscript. All authors have read and approved the final manuscript.

\section{Ethics approval and consent to participate}

Not applicable.

\section{Patient consent for publication}

Not applicable. 


\section{Competing interests}

The authors declare that they have no competing interests.

\section{References}

1. Neri M, Riezzo I, Pascale N, Pomara C and Turillazzi E: Ischemia/reperfusion injury following acute myocardial infarction: A critical issue for clinicians and forensic pathologists. Mediators Inflamm 2017: 7018393, 2017.

2. Li P, Stetler RA, Leak RK, Shi Y, Li Y, Yu W, Bennett MVL and Chen J: Oxidative stress and DNA damage after cerebral ischemia: Potential therapeutic targets to repair the genome and improve stroke recovery. Neuropharmacology 134: 208-217, 2018.

3. Cheng YC, Sheen JM, Hu WL and Hung YC: Polyphenols and oxidative stress in atherosclerosis-related ischemic heart disease and stroke. Oxid Med Cell Longev 2017: 8526438, 2017.

4. Li YQ, Zhan ZL and Li QF: Exploration of protective effect of Sevoflurane preconditioning on hypoxia reoxygenation injury of myocardial cells in rats and related molecular mechanisms. J Hainan Med Univ 22: 8-11, 2016.

5. Wen T, Wang L, Sun XJ, Zhao X, Zhang GW and Ling LJ: Sevoflurane preconditioning promotes activation of resident CSCs by transplanted BMSCs via miR-210 in a rat model for myocardial infarction. Oncotarget 8: 114637-114647, 2017.

6. Pagel PS and Crystal GJ: The discovery of myocardial preconditioning using volatile anesthetics: A history and contemporary clinical perspective. J Cardiothorac Vasc Anesth 32: 1112-1134, 2018.

7. Xie D, Zhao J, Guo R, Jiao L, Zhang Y, Lau WB, Lopez B, Christopher T, Gao E, Cao J, et al: Sevoflurane pre-conditioning ameliorates diabetic myocardial ischemia/reperfusion injury via differential regulation of p38 and ERK. Sci Rep 10: 23, 2020.

8. Kunst G and Klein AA: Peri-operative anaesthetic myocardial preconditioning and protection - cellular mechanisms and clinical relevance in cardiac anaesthesia. Anaesthesia 70: 467-482, 2015.

9. Zhang SB, Liu TJ, Pu GH, Li BY, Gao XZ and Han XL: MicroRNA-374 exerts protective effects by inhibiting SP1 through activating the PI3K/Akt pathway in rat models of myocardial ischemia-reperfusion after sevoflurane preconditioning. Cell Physiol Biochem 46: 1455-1470, 2018.

10. Zhang Y, Huang J, Yang X, Sun X, Xu Q, Wang B, Zhong P and Wei X: Altered Expression of TXNIP in the peripheral leukocytes of patients with coronary atherosclerotic heart disease. Medicine (Baltimore) 96: e9108, 2017.

11. Wang $\mathrm{CY}, \mathrm{Xu} \mathrm{Y}$, Wang $\mathrm{X}$, Guo $\mathrm{C}$, Wang $\mathrm{T}$ and Wang $\mathrm{ZY}$ : Dl-3-n-butylphthalide inhibits NLRP3 inflammasome and mitigates alzheimer's-Like pathology via Nrf2-TXNIP-TrX axis. Antioxid Redox Signal 30: 1411-1431, 2019.

12. Friedemann T, Schumacher U, Tao Y, Leung AK and Schröder S: Neuroprotective activity of coptisine from coptis chinensis (Franch). Evid Based Complement Alternat Med 2015: 827308, 2015.

13. Ye X, Zuo D, Yu L, Zhang L, Tang J, Cui C, Bao L, Zan K, Zhang Z, Yang X, et al: ROS/TXNIP pathway contributes to thrombin induced NLRP3 inflammasome activation and cell apoptosis in microglia. Biochem Biophys Res Commun 485: 499-505, 2017.

14. Ji L, Wang Q, Huang F, An T, Guo F, Zhao Y, Liu Y, He Y, Song Y and Qin G: FOXO1 overexpression attenuates tubulointerstitial fibrosis and apoptosis in diabetic kidneys by ameliorating oxidative injury via TXNIP-TRX. Oxid Med Cell Longev 2019: 3286928, 2019.

15. Han X, Wu YC, Meng M, Sun QS, Gao SM and Sun H: Linarin prevents LPSinduced acute lung injury by suppressing oxidative stress and inflammation via inhibition of TXNIP/NLRP3 and NFKB pathways. Int J Mol Med 42: 1460-1472, 2018.

16. Chen J, Hui ST, Couto FM, Mungrue IN, Davis DB, Attie AD, Lusis AJ, Davis RA and Shalev A: Thioredoxin-interacting protein deficiency induces Akt/Bcl-xL signaling and pancreatic beta-cell mass and protects against diabetes. FASEB J 22: 3581-3594, 2008.

17. Narita Y, Nagane M, Mishima K, Huang HJ, Furnari FB and Cavenee WK: Mutant epidermal growth factor receptor signaling down-regulates p27 through activation of the phosphatidylinositol 3-kinase/Akt pathway in glioblastomas. Cancer Res 62: 6764-6769, 2002.
18. Oyagbemi AA, Omobowale TO, Asenuga ER, Ochigbo GO, Adejumobi AO, Adedapo AA and Yakubu MA: Sodium arsenite-induced cardiovascular and renal dysfunction in rat via oxidative stress and protein kinase B (Akt/PKB) signaling pathway. Redox Rep 22: 467-477, 2017.

19. Cui Q, Li X and Zhu H: Curcumin ameliorates dopaminergic neuronal oxidative damage via activation of the Akt/Nrf2 pathway. Mol Med Rep 13: 1381-1388, 2016.

20. Wang L, Shen S, Xiao H, Ding F, Wang M, Li G and Hu F: ARHGAP24 inhibits cell proliferation and cell cycle progression and induces apoptosis of lung cancer via a STAT6-WWP2-P27 axis. Carcinogenesis (bjz144): Aug 20, 2019 (Epub ahead of print).

21. Wang $\mathrm{Z}, \mathrm{Yu} \mathrm{C}$ and Wang H: HOXA5 inhibits the proliferation and induces the apoptosis of cervical cancer cells via regulation of protein kinase B and p27. Oncol Rep 41: 1122-1130, 2019.

22. Williams V, Brichler S, Khan E, Chami M, Dény P, Kremsdorf D and Gordien E: Large hepatitis delta antigen activates STAT-3 and NF- $\kappa B$ via oxidative stress. J Viral Hepat 19: 744-753, 2012.

23. Smith RS Jr, Agata J, Xia CF, Chao L and Chao J: Human endothelial nitric oxide synthase gene delivery protects against cardiac remodeling and reduces oxidative stress after myocardial infarction. Life Sci 76: 2457-2471, 2005.

24. Zhao X, Qi H, Zhou J, Xu S and Gao Y: P27 protects cardiomyocytes from sepsis via activation of autophagy and inhibition of apoptosis. Med Sci Monit 24: 8565-8576, 2018.

25. Ma L, Liu H, Xie Z, Yang S, Xu W, Hou J and Yu B: Ginsenoside $\mathrm{Rb} 3$ protects cardiomyocytes against ischemia-reperfusion injury via the inhibition of JNK-mediated NF-kappaB pathway: A mouse cardiomyocyte model. PLoS One 9: e103628, 2014.

26. Kim DE, Kim B, Shin HS, Kwon HJ and Park ES: The protective effect of hispidin against hydrogen peroxide-induced apoptosis in $\mathrm{H} 9 \mathrm{c} 2$ cardiomyoblast cells through Akt/GSK-3 $\beta$ and ERK1/2 signaling pathway. Exp Cell Res 327: 264-275, 2014.

27. Jun HO, Kim DH, Lee SW, Lee SH, Seo JH, Kim JH, Kim JH, Yu YS, Min BH and Kim KW: Clusterin protects H9c2 cardiomyocytes from oxidative stress-induced apoptosis via Akt/GSK-3 $\beta$ signaling pathway. Exp Mol Med 43: 53-61, 2011.

28. Zhai M, Li B, Duan W, Jing L, Zhang B, Zhang M, Yu L, Liu Z, Yu B, Ren K, et al: Melatonin ameliorates myocardial ischemia reperfusion injury through SIRT 3-dependent regulation of oxidative stress and apoptosis. J Pineal Res 63: e12419, 2017.

29. Zhao D, Yang J and Yang L: Insights for oxidative stress and mTOR signaling in myocardial ischemia/reperfusion injury under diabetes. Oxid Med Cell Longev 2017: 6437467, 2017.

30. Yu L, Li Q, Yu B, Yang Y, Jin Z, Duan W, Zhao G, Zhai M, Liu L, Yi D, et al: Berberine attenuates myocardial ischemia/reperfusion injury by reducing oxidative stress and inflammation response: Role of silent information regulator 1. Oxid Med Cell Longev 2016: 1689602, 2016.

31. Qian B, Yang Y, Yao Y, Liao Y and Lin Y: Upregulation of vascular endothelial growth factor receptor-1 contributes to sevoflurane preconditioning-mediated cardioprotection. Drug Des Devel Ther 12: 769-776, 2018.

32. Pasqualin RC, Mostarda CT, de Souza LE, Vane MF, Sirvente R, Otsuki DA, Torres MLA, Irigoyen MCC and Auler JOC Jr: Sevoflurane preconditioning during myocardial ischemia-reperfusion reduces infarct size and preserves autonomic control of circulation in rats. Acta Cir Bras 31: 338-345, 2016.

33. Wang S, Zhao X, Yang S, Chen B and Shi J: Salidroside alleviates high glucose-induced oxidative stress and extracellular matrix accumulation in rat glomerular mesangial cells by the TXNIP-NLRP3 inflammasome pathway. Chem Biol Interact 278: 48-53, 2017.

34. Hou X, Yang S and Yin J: Blocking the REDD1/TXNIP axis ameliorates LPS-induced vascular endothelial cell injury through repressing oxidative stress and apoptosis. Am J Physiol Cell Physiol 316: C104-C110, 2019.

35. Yao YL, Yang X, Xue XW, Fan LF and Jiao XY: Effect of adenovirus-mediated TXNIP overexpression on apoptosis and injury of H9C2 cardiomyocytes. Sheng Li Xue Bao 65: 309-318, 2013 (In Chinese).

36. Petrenko AB, Yamakura T, Sakimura K and Baba H: Defining the role of NMDA receptors in anesthesia: Are we there yet? Eur J Pharmacol 723: 29-37, 2014.

37. Yamaguchi F, Hirata Y, Akram H, Kamitori K, Dong Y, Sui L and Tokuda M: FOXO/TXNIP pathway is involved in the suppression of hepatocellular carcinoma growth by glutamate antagonist MK-801. BMC cancer 13: 468, 2013. 
38. Papadia S, Soriano FX, Léveillé F, Martel M-A, Dakin KA, Hansen HH, Kaindl A, Sifringer M, Fowler J, Stefovska V, et al Synaptic NMDA receptor activity boosts intrinsic antioxidant defenses. Nature neuroscience 11: 476-487, 2008.

39. Mahmoud AM, Wilkinson FL, McCarthy EM, Moreno-Martinez D, Langford-Smith A, Romero M, Duarte J and Alexander MY: Endothelial microparticles prevent lipid-induced endothelial damage via Akt/eNOS signaling and reduced oxidative stress. FASEB J 31: 4636-4648, 2017.
40. Zeng B, Liu L, Liao X, Zhang $\mathrm{C}$ and Ruan H: Thyroid hormone protects cardiomyocytes from $\mathrm{H}_{2} \mathrm{O}_{2}$-induced oxidative stress via the PI3K-AKT signaling pathway. Exp Cell Res 380: 205-215, 2019.

41. Hussien-Ali A and Alifragis P: P27 Minimising the toxic effects of beta-Amyloid 42 on oxidative stress. Biochem Pharmacol 139: 133-134, 2017. 